\title{
Births Averted by Induced Abortion: An Application of Renewal Theory
}

\author{
R. G. Potter \\ Department of Sociology, Brown University Providence, Rhode Island 02912 \\ and Population Studies Center, University of Michigan, Ann Arbor, Michigan 48104
}

Received February 15, 1971

\section{INTRODUCTION}

It is increasingly recognized that few if any populations have undergone the transition from high to low fertility without significant recourse to induced abortion (Davis, 1963; Kirk, 1967). The strategic role played by induced abortion in helping to bring down the birth rate of Japan and the nations of Eastern Europe is well documented (Muramatsu, 1960; Hall, 1970). That its contribution to family planning in the United States will increase is made likely by the legal changes now occurring in several states as well as recent advances in abortion technique. Yet despite the importance of the topic, a mathematical theory of induced abortion and its impact on natality has not been previously developed.

For two reasons, an induced abortion cannot be equated with preventing a birth. First, the effect of a particular abortion is conditional upon the potential outcome of the pregnancy it terminates. If the pregnancy might have ended in miscarriage, then essentially nothing is gained; if the pregnancy might have ended in a stillbirth, far from gaining protection, several months of pregnancy and anovulation are being forfeited. Only when the aborted pregnancy might have produced a live birth does an appreciable delay of the next birth ensue.

Second, even when the pregnancy aborted was otherwise fated to end in a live birth, that abortion is typically not equivalent to averting a live birth. Months of gestation and anovulation associated with an abortion are fewer than those associated with a live birth. Therefore a woman is returned to fecundable status sooner after an abortion than a live birth.

As a result, it is not obvious whether 100 induced abortions are averting 75,50 , or more nearly 35 births. Moreover, one would like to know the manner in which the impact per abortion varies according to the age and fecundity of the woman, the timing of her abortion as measured in terms of missed menstrual periods, and the efficiency of her accompanying contraception.

Previous theoretical work (Potter, 1963; Sheps, 1964; Srinivasan, 1967) has

(C) 1972 by Academic Press, Inc. 
considered changes in risk of early fetal wastage, but has not addressed directly the issue of births averted by abortion. The present treatment does so by comparing pairs of cohorts alike in all respects except that one cohort of women uses induced abortion while the other does not. Through an extension of earlier work (Potter 1970a, 1970b), each cohort is formulated as a renewal process.

It will be shown that four factors are conducive to a higher impact per abortion. First is a lower risk of spontaneous fetal wastage, tending to reserve a higher proportion of abortions for pregnancies otherwise fated to end in live births. Second is a later timing of abortion, resulting in a higher fraction of miscarriages occurring early enough to forestall an otherwise wasted abortion. Third is a shorter length of postpartum anovulation, tending to reduce the differential in gestational and anovulatory months between a live birth and an abortion. Fourth, and most important, is a longer fecundable period per conception. When the expected number of fecundable months per conception are few-say, 6-8 as might be expected in the absence of contraception-then an abortion is worth less than 0.5 births averted. In contrast, when the expected fecundable period per conception is long by virtue of efficient contraception, then an induced abortion may be equivalent to more than 0.8 births averted.

In recognition that the assumptions of the model are highly simplified, the last section of the paper compares results from the present model with those of a more elaborate model that encompasses secondary sterility as well as agedependent declines in other aspects of fecundity. The derivation of formulas is relegated to two appendices.

\section{NOMENClature}

The nutation of the present article is very close to an earlier onc (Pottcr, 1970b). Nevertheless, for purposes of review and to note the few changes and additions, a glossary of terms is provided.

$S_{0} \quad$ State of being fecundable and subject to a risk of conception

$S_{1} \quad$ Pregnant

$S_{2} \quad$ Infecundable owing to the temporary anovulation period that follows abortion or miscarriage

$S_{3} \quad$ Anovulatory following a stillbirth

$S_{4} \quad$ Anovulatory following a live birth

$T_{i j} \quad$ Random time required for passage from state $S_{i}$ to $S_{j}$

$T_{i j}^{*} \quad$ Same random time when $S_{j}$ is the next state entered after $S_{i}$, $i, j=0,1,2,3,4$ 

$u_{i j}, u_{i j}^{*} \quad$ Corresponding mean passage times (Since $u_{01}=u_{01}^{*}$ and $u_{i 0}=u_{i 0}^{*}$, $i=2,3,4$ invariably, the asterisk will be omitted.)
$\theta_{i} \quad$ Probability of pregnancy ending in outcome $i$

$$
\begin{aligned}
i & =2 \text { miscarriage } \\
i & =3 \text { stillbirth } \\
i & =4 \text { live birth }
\end{aligned}
$$
$\eta_{i} \quad u_{1 i}^{*}+u_{i 0}$, mean infecundable period (gestation plus postdelivery anovulation) associated with a pregnancy of outcome $i$

$E\left[N_{i}(T) \mid S_{j}\right]$ Expected number of events $i$ during a duration $T$ if the process starts in state $S_{j}$

$f \quad$ Natural fecundability

$e \quad$ Effectiveness of contraception

$p \quad(1-e) f$, the monthly risk of conception during a fecundable month when practicing contraception

$d^{\prime} \quad$ Monthly probability of discontinuing contraception during an anovulatory month

$d \quad(1-p) d^{\prime}$, monthly probability of discontinuing contraception for a reason other than accidental pregnancy during a fecundable month

$u_{01, i} \quad$ Expected delay to next conception if contraception is initiated right after a pregnancy of outcome $i$

$u_{01,0} \quad$ Expected delay to next conception if contraception is initiated at start of the first fecundable month following anovulation

$Z \quad$ Duration in months from last menstrual period to induced abortion

$h \quad$ Proportion of miscarriages occurring carly enough to forestall an induced abortion

$H \quad 1 /\left(1-\theta_{2} h\right)$

$\delta$

Mean difference in pregnancy length between miscarriage occuring early enough to forestall abortion and miscarriage in the absence of abortion

$I \quad$ Net delay of next birth per induced abortion

$B \quad$ Births averted per induced abortion

$\phi(i) \quad$ Proportion of miscarriages lasting $i$ months from last menstrual period in the absence of induced abortion 


\section{MODEL}

'To measure births averted per induced abortion, two cohorts of women identical except that one uses abortion and the other does not are compared over a residual reproductive period that lasts $T$ months and starts with a live birth (the "origin birth"). Mathematically, each cohort is represented by a renewal process. The conceptual experiments are of two types. In the "single abortion experiment," the control cohort practices the same contraceptive repetitively each successive pregnancy interval of the residual reproductive period, but practices no induced abortion. The experimental cohort, possessed of the same fecundity and practicing the same contraceptive repetitively each pregnancy interval, differs from the control cohort only in that its members each deliberately abort the first accidental pregnancy that does not end in early miscarriage but thereafter make no further use of abortion. By virtue of this single abortion, the next birth is delayed, thereby shortening the subsequent period when additional excess births are a possibility. The difference in mean interval from origin birth to next birth between cxpcrimental and control cohort measures the net delay of next birth per abortion. The difference between cohorts in mean births per woman at the end of the residual reproductive period defines births averted per induced abortion.

This approach by single abortion experiments has the advantage of parallelism with the approach used already (Potter 1970a, 1970b) to measure the net delay of next conception and births averted per segment of contraception. However, women who resort to induced abortion once are likely to do so again if they experience another pregnancy. Accordingly, from an applied point of view, it is useful to consider "repeated abortion experiments" as well. The control cohort is the same as before, its members practicing contraception each pregnancy interval without recourse to induced abortion. The experimental cohort is identical to the control cohort in all respects except that its members deliberately abort every accidental pregnancy that does not end in early miscarriage. For this case there is no measure of net delay of next birth per abortion since in the experimental cohort no births are allowed. The mean number of births per woman of the control cohort divided by the mean number of induced abortions per woman of the experimental cohort yields a measure of births averted per abortion. It will be shown (Appendix II) that in the limit, as $T$ is allowed to become very large, the number of births averted per abortion in a repeatedabortion cxpcriment converges to the number averted in the corresponding single-abortion experiment. This means that in the results section below which is restricted to a consideration of births averted per abortion and its correlates, no distinction need be made between the two types of experiment.

As regards a single abortion experiment, to qualify as a renewal process, the present model must generate birth intervals that behave as independent, identi- 
cally distributed random variables except for the initial birth interval which may be distributed independently of them. For purposes of the repeated abortion experiment, the birth intervals of the control cohort and the pregnancy intervals of the experimental cohort must behave analogously.

To meet these specifications, it is convenient to make the following simplifying assumptions:

(1) homogeneity among women (the same probabilities and duration of residual reproductive period apply to all women);

(2) homogeneity in time (all parameters are fixed in time and therefore are independent of age);

(3) the residual reproductive period is "sufficiently long", i.e., infinite.

The above homogeneity assumptions apply to contraceptive efficiency as well as to fecundity.

Several additional assumptions are not necessary to generate a renewal process, but greatly simplify the algebra while exercising only minor effects on results.

(4) Conditional to outcome of pregnancy, the period of temporary anovulation following delivery is constant, being $u_{20}$ following a miscarriage, $u_{30}$ following a stillbirth and $u_{40}$ following a live birth.

(5) Gestation preceding a stillbirth is of constant length $u_{13}^{*}$; that preceding a live birth is constant at $u_{14}^{*}$; while pregnancy length preceding miscarriage follows a truncated geometric distribution (defined in Appendix I).

These last two assumptions mean that for a given outcome of pregnancy, anovulatory and gestational lengths are independently distributed. Furthermore, combined with assumptions (1), (2), and (3) they assure the independence of conceptive waiting times $T_{01}$ and infecundable periods $T_{1 i}^{*}+T_{i 0}$.

(6) The time unit is one month and an arbitrary order is imposed upon events within a month. Contraception is initiated or resumed always near the start of a month; ovulation and therefore the possibility of conception occurs in the middle of the month; and contraception is discontinued near the end of the month. Termination of pregnancy also occurs near the end of the month.

As a consequence of (6), most months may be classed unambiguously as a month of gestation, a month of anovulation, or as a fecundable month. The exception is months encompassing conception. These latter are classed as months of gestation with the understanding that pregnancy duration is measured from the last menstrual period. It follows that any waiting time $T_{01}$ consists solely of fecundable months.

The following assumptions concern induced abortion.

(7) The temporary anovulatory period following induced abortion is the same as that following miscarriage. 
(8) In a given abortion experiment, all induced abortions are performed with the same timing, namely, $Z$ months after the last menstrual period (LMP), $Z=2,3, \ldots, 7$.

(9) The timing $Z$ of abortion determines the fraction $h$ of miscarriages occurring early enough to forestall abortion:

$$
\begin{aligned}
h & =\frac{\phi(2)}{2} \quad \text { when } & Z=2, \\
& =\sum_{i=2}^{Z-1} \phi(i)+\frac{\phi(Z)}{2} & Z=3, \ldots, 7,
\end{aligned}
$$

where $\phi(i)$ is the proportion of miscarriages that in the absence of induced abortion would be expected to occur at the end of the $i$-th month from LMP, $i-2,3, \ldots, 7$. It is assumed that among the $\phi(Z)$ miscarriages potentially coinciding with the induced abortion at time $Z$, one half show symptoms far enough in advance to forestall the abortion while the other half fail to avert an unnecessary operation. All miscarriages occurring during months carlicr than $Z$ anticipate an abortion.

(10) Done with medical competence, the induced abortion does not lower fecundity.

Potentially the impact of abortion could be quite large if the operations were not only terminating the immediate pregnancy, but reducing subsequent fecundity as well.

\section{RESULTS}

Basic results, derived in Appendix I for single-abortion experiments and in Appendix II for repeated abortion experiments, are

$$
B=I / u_{44},
$$

where

$$
\begin{aligned}
u_{44} & =\frac{1}{\theta_{4}}\left\{\sum_{i=2}^{4} \theta_{i}\left(u_{01, i}+\eta_{i}\right)\right\}, \\
I & =H\left(u_{01,2}+\eta_{2}\right)-(H-1) \delta+\left(Z-u_{12}^{*}\right), \\
u_{01, i} & =k u_{01,0}+(1-k)\left(\frac{1-f}{f}\right), \\
k & =\left(1-d^{\prime}\right)^{u_{i 0}}, \\
u_{01,0} & =\frac{1-p}{d+p}+\frac{d}{d+p}\left(\frac{1-f}{f}\right) .
\end{aligned}
$$


Typically the expected conceptive delays

$$
u_{01,4} \leqslant u_{01,3} \leqslant u_{01,2} \leqslant u_{01,0}
$$

are within a few months of each other, the differences decreasing to zero as $e \rightarrow 0.0$ or $d \rightarrow 0.0$.

As will be shown below, the term $(H-1) \delta$ is typically small enough to ignore. If $Z$ is set at 3 , the term $Z-u_{12}^{*}$ may also be neglected and to a very good approximation

$$
I=H\left(u_{01,2}+\eta_{2}\right)
$$

In the context of repeated abortion, $H\left(u_{01,2}+\eta_{2}\right)$ may be interpreted as the recurrence time between induced abortions, $H$ being the number of pregnancies (including early miscarriages) per induced abortion.

If we assume that contraception is absent, equivalent to setting $e=0.0$ and therefore obtaining $p=f$, then the formula for births averted per abortion simplifies to

$$
B=\frac{H\left(\frac{1-f}{f}+\eta_{2}\right)}{\frac{1}{\theta_{4}}\left(\frac{1-f}{f}+\sum_{i=2}^{4} \theta_{i} \eta_{i}\right)} .
$$

In this case it may be seen that, other factors constant, the impact of abortion is higher:

(a) the lower is the risk of spontaneous fetal wastage (and therefore the closer is $\theta_{4}$ to 1.0 ),

(b) the lower is natural fecundability $f$,

(c) the shorter is postpartum anovulation $u_{40}=\eta_{4}-u_{14}^{*}$, and

(d) the larger is $Z$ (increasing $h$ and hence $H$ ).

However, in the presence of efficient contraception, the conceptive delays $u_{01, i}$ come to dominate both the numerator $I$ and the denominator $u_{\mathbf{4}}$ to give a ratio fairly close to 1.0. In the limit (cf. Appendix I), if we let the conceptive delay terms become very large, $B$ approaches $\theta_{4} H$, the maximum impact per abortion compatible with a given set of fecundity parameters.

When a woman ages, her fecundity declines. This decline has three dimensions which together exercise a mixed effect on the impact of induced abortion. If we label the components of fecundity decline + or - according as their effect on births averted per abortion is positive or negative, we have:

1. declining fecundability $(+)$,

2. increasing risk of spontaneous fetal wastage $(-)$,

3. increasing length of postpartum anovulation $(-)$. 
To generalize about the relative sensitivity of abortion impact to the several variables contained in the present model presupposes knowing the empirical ranges of these variables. Having by far the largest range of empirical value are the conceptive delays $u_{01, i}$. In the absence of contraception, the $u_{01, i}$ typically vary from 5 to 12 months, mainly as a function of the woman's age; but in the presence of efficient contraception they may assume very large magnitudes. The empirical range of postpartum anovulation $u_{40}$ is 3-13 months, relatively much larger than the ranges for $u_{20}$ or $u_{30}$. Ranges of the expected pregnancy lengths $u_{1 i}^{*}$ are also small. Typical values for $\theta_{2}$ are $.10-.20$, though ranging up to .30 late in the reproductive period. Values of $\theta_{3}$ usually fall within a span of .01-.06.

Most induced abortions are timed within a $Z$-range of 2-4. Until a menstrual period is missed, a woman lacks definite evidence of pregnancy. An "early" abortion $(Z=2), 6$ weeks after conception or 8 weeks after LMP, implies but one missed menstrual period. Nor is an abortion likely to be later than the fourth month (implying three missed periods) since then it involves more difficult and risky medical procedures.

From this specification of ranges it appcars that the most crucial factor in determining births averted per abortion is the efficiency of accompanying contraception (measured by parameters $e$ and $d$ ). The impact of abortion is more secondarily sensitive to the timing of abortion $Z$, risk of miscarriage $\theta_{2}$, and

TABLE I

Fecundity Parameters and Associated Birth Interval, by Age of Woman, Assuming No Contraception or Induced Abortion

\begin{tabular}{lcccc}
\hline & \multicolumn{5}{c}{ Age of woman } \\
\cline { 2 - 5 } Parameter $^{c}$ & 27.5 & 32.5 & 37.5 & 42.5 \\
\hline $1 / f=u_{01}+1$ & 8.0 & 9.0 & 10.0 & 11.0 \\
$\theta_{2}$ & 0.085 & 0.119 & 0.170 & 0.230 \\
$\theta_{3}=(0.15 / 0.85) \theta_{2}$ & 0.015 & 0.021 & 0.030 & 0.041 \\
$u_{14}^{*}$ & 10.0 & 10.0 & 10.0 & 10.0 \\
$u_{13}^{*}$ & 9.0 & 9.0 & 9.0 & 9.0 \\
$u_{12}^{*}$ & 3.0 & 3.0 & 3.0 & 3.0 \\
$u_{40}^{*}$ & 10.0 & 12.0 & 13.0 & 13.0 \\
$u_{30}$ & 2.0 & 2.0 & 2.0 & 2.0 \\
$u_{20}$ & 1.0 & 1.0 & 1.0 & 1.0 \\
$u_{44}{ }^{b}$ & 28.4 & 32.4 & 35.6 & 38.6 \\
\hline
\end{tabular}

${ }^{a}$ In the absence of contraception (i.e., $e=0.0$ ), $u_{01, i}=u_{01,0}=(1-f) / f, i=2,3,4$.

${ }^{b}$ Based on the formula $u_{44}=1 / \theta_{4}\left\{(1-f) / f+\sum_{i=2}^{4} \theta_{i} \eta_{i}\right\}$. 
TABLE II

Functions of the Timing of Abortion $Z$

\begin{tabular}{cccccccc}
\hline$Z$ & & & & \multicolumn{2}{c}{$\theta_{2}=0.10$} & \multicolumn{1}{c}{$\theta_{2}=0.30$} \\
\cline { 5 - 8 } & $h$ & $\begin{array}{c}Z-u_{12}^{* a} \\
\text { (months) }\end{array}$ & $\begin{array}{c}\delta \\
\text { (months) }\end{array}$ & $\begin{array}{c}(H-1) \delta \\
\text { (months) }\end{array}$ & $H$ & $\begin{array}{c}(H-1) \delta \\
\text { (months) }\end{array}$ \\
\hline 2 & 0.23 & -1.12 & 1.12 & 1.024 & 0.03 & 1.075 & 0.08 \\
3 & 0.59 & -0.12 & 0.90 & 1.062 & 0.06 & 1.211 & 0.19 \\
4 & 0.79 & 0.88 & 0.62 & 1.085 & 0.05 & 1.305 & 0.19 \\
\hline
\end{tabular}

${ }^{a}$ Computed to two-place accuracy, $u_{12}^{*}=3.12$. Cf. Appendix II.

natural fecundability $f$. The importance of $Z$ increases as $\theta_{2}$ increases. Only when contraception is absent or inefficient as reflected in modest $u_{01, i}$ values does the expected length of postpartum anovulation $u_{\mathbf{4 0}}$ become important.

For illustration, a set of age-specific parameter assignments are assembled in Table I. These assignments are taken from an earlier analysis (Potter, 1971), which rests heavily on a series of secondary analyses of Taiwan data (Jain, 1969a, 1969b, 1970).

The behavior of several functions of $Z$ that are important to the value of $I$ are cxhibitcd in Table II as $Z$ varies from 2 to 4 . The trival size of $(H-1) \delta$ is manifest. Evan when $\theta_{2}=0.30$, an extreme value, the term in question has a value less than .25 (in months). The term $\left(Z-u_{12}^{*}\right)$ varies roughly as $-1,0$, and 1. Reflecting mainly the effect of $H$, late abortion $(Z=4)$ as compared to

TABLE III

Births Averted per Abortion, by Wife's Starting Age and Efficiency of Contraception ${ }^{a}$

\begin{tabular}{|c|c|c|c|c|c|c|c|c|}
\hline \multirow{3}{*}{$\begin{array}{l}\text { Wife's age } \\
\text { at start of } \\
\text { family } \\
\text { limitation }\end{array}$} & \multicolumn{5}{|c|}{ Traditional contraception $\left(d^{\prime}=0.0\right)$} & \multicolumn{3}{|c|}{$\begin{array}{c}\text { Modern contraception } \\
\qquad(e=1.0)\end{array}$} \\
\hline & \multicolumn{5}{|c|}{ Effectiveness $e$} & \multicolumn{3}{|c|}{ Discontinuation rate $d^{\prime}$} \\
\hline & 0.00 & 0.75 & 0.90 & 0.95 & 0.98 & 0.05612 & 0.02846 & 0.01191 \\
\hline 27.5 & 0.41 & 0.67 & 0.81 & 0.87 & 0.91 & 0.73 & 0.81 & 0.88 \\
\hline 32.5 & 0.40 & 0.66 & 0.79 & 0.85 & 0.89 & 0.71 & 0.79 & 0.86 \\
\hline 37.5 & 0.41 & 0.65 & 0.77 & 0.83 & 0.86 & 0.69 & 0.77 & 0.83 \\
\hline 42.5 & 0.42 & 0.62 & 0.72 & 0.77 & 0.79 & 0.67 & 0.74 & 0.79 \\
\hline
\end{tabular}

a Based on $B=H\left(u_{01,2}+4.0\right) / u_{44}$. 
early abortion $(Z=2)$ increases the value of $I$ about $5 \%$ when $\theta_{2}=0.10$, but considerably more than that when $\theta_{2}=0.30$.

Births averted per induced abortion depends primarily on the efficiency of the contraception being backstopped and is rather insensitive to age. These two relationships are illustrated by Table III. In this Table the age-specific fecundity values of Table I are stipulated together with $Z=3$. Failure of traditional contraceptives such as condom or diaphragm and jelly to prevent accidental pregnancy is attributable primarily to irregularity of practice. To represent this situation, we fix $\mathrm{d}^{\prime}=0.0$, but vary $e$ in order to represent different degrees of regularity of practice.

As regards modern contraception (mainly IUD and otal contraception), the principal limiting factor is continuation. Accordingly, perfect effectiveness is assumed (i.e., $e=1.0$ ), but the parameter $d^{\prime}$ is varied. The values $d^{\prime}=.05612$, .02846 , and .01191 correspond to 25,50 , and $75 \%$ of users continuing as long as two years. In Korea, Malaysia, and Taiwan, the proportion of acceptors retaining their first intrauterine device for two years has been in the vicinity of .5 while continuation of oral contraception has been appreciably less (Mauldin et al., 1967; Jones and Mauldin, 1967).

\section{A Test}

Throughout the paper a constant fecundity combined with a very long reproductive period is assumed. In reality fecundity declines progressively during the middle and later stages of the reproductive period while secondary sterility imposes a variable and limited reproductive length. Accordingly the results reported in Table III remain suspect until tested by a model elaborate enough to include the agedependent decline in fecundity and secondary sterility.

Fortunately a limited set of runs are available from such a model. ACCOFERT (Potter, 1971) is a computerized model, based on matrix multiplication with nonstationary transition probabilitics. Secondary sterility is included as an absorbing state. The model is predicated on essentially the same set of fecundity assumptions as contained in Table I. Repeated abortion experiments are possible with ACCOFERT, but not single abortion experinents.

A set of results corresponding to those of Table III are given in Table IV. Exact correspondence cannot be expected owing to a number of differences in the two sets of assumptions. The most important are that ACCOFERT

(1) allows for secondary sterility,

(2) provides that with advancing age, fecundability $f$ declines, the risks of spontaneous fetal loss $\theta_{2}$ and $\theta_{3}$ rise, and the expected length of postpartum anovulation $u_{40}$ increases, 
(3) the anovulatory period following a stillbirth or live birth is variable rather than constant in length,

(4) gestational length preceding miscarriage or abortion is constant (at 3 months) rather than variable,

(5) the parameter $h$ is fixed at 0.50 .

\section{TABLE IV}

Births Averted per Abortion, by Wife's Starting Age and Efficiency of Contraception, Based on the Cohort Model ACCOFERT

\begin{tabular}{|c|c|c|c|c|c|c|c|c|}
\hline \multirow{3}{*}{$\begin{array}{l}\text { Wife's age } \\
\text { at start of } \\
\text { family } \\
\text { limitation }\end{array}$} & \multicolumn{5}{|c|}{ Traditional contraception $\left(d^{\prime}=0.0\right)$} & \multicolumn{3}{|c|}{$\begin{array}{l}\text { Modern contraceptives } \\
\qquad(e=1.0)\end{array}$} \\
\hline & \multicolumn{5}{|c|}{ Effectiveness $e$} & \multicolumn{3}{|c|}{ Discontinuation rate $d^{\prime}$} \\
\hline & 0.00 & 0.75 & 0.90 & 0.95 & 0.98 & 0.05612 & 0.02846 & 0.01191 \\
\hline 27.5 & 0.43 & 0.68 & 0.78 & 0.84 & 0.84 & 0.70 & 0.78 & 0.83 \\
\hline 32.5 & 0.43 & 0.67 & 0.77 & 0.82 & 0.85 & 0.68 & 0.75 & 0.81 \\
\hline 37.5 & 0.46 & 0.68 & 0.76 & 0.80 & 0.82 & 0.68 & 0.74 & 0.79 \\
\hline 42.5 & 0.56 & 0.72 & 0.77 & 0.79 & 0.79 & 0.72 & 0.76 & 0.78 \\
\hline
\end{tabular}

TABLE V

Relative Differences in Impact per Abortion Between ACCOFERT and Present Model, by Wife's Starting Age and Efficiency of Contraception ${ }^{a}$

\begin{tabular}{|c|c|c|c|c|c|c|c|c|}
\hline \multirow{3}{*}{$\begin{array}{l}\text { Wife's age } \\
\text { at start of } \\
\text { family } \\
\text { limitation }\end{array}$} & \multicolumn{5}{|c|}{ Traditional contraception $\left(d^{\prime}=0.0\right)$} & \multicolumn{3}{|c|}{$\begin{array}{l}\text { Modern contraception } \\
\qquad(e=1.0)\end{array}$} \\
\hline & \multicolumn{5}{|c|}{ Effectiveness $e$} & \multicolumn{3}{|c|}{ Discontinuation rate $d^{\prime}$} \\
\hline & 0.00 & 0.75 & 0.90 & 0.95 & 0.98 & 0.05612 & 0.02846 & 0.01191 \\
\hline 27.5 & -0.06 & 0.00 & 0.03 & 0.03 & 0.08 & 0.04 & 0.05 & 0.06 \\
\hline 32.5 & -0.09 & -0.01 & 0.02 & 0.04 & 0.05 & 0.04 & 0.05 & 0.06 \\
\hline 37.5 & -0.14 & -0.04 & 0.01 & 0.03 & 0.04 & 0.01 & 0.03 & 0.05 \\
\hline 42.5 & -0.33 & -0.15 & -0.06 & -0.03 & -0.01 & -0.09 & -0.03 & 0.02 \\
\hline
\end{tabular}

${ }^{a}$ Table values are computed by $\left(B_{1}-B_{2}\right) / B_{1}$ where $B_{1}$ and $B_{2}$ denote births averted per induced abortion (to 4 decimal places) as estimated by present model and ACCOFERT, respectively. 
Despite this list of differences, examination of Tables III and IV reveal rather similar magnitudes. Both models show that for a given starting age, impact per abortion increases as the efficiency of contraception increases.

At the same time two biases emerge (Table V) when differences in corresponding entries of Tables III and IV are expressed as ratios of the entries of Table III. First is a tendency for the present model to understate the impact of abortion in a situation of no or inefficient contraception combined with a late starting age. It is hypothesized that this bias arises from the neglect of secondary sterility. If secondary sterility intervenes before another birth is possible, it ceases to matter that a woman returns to fecundable status a few months sooner after the abortion than after the live birth it has prevented.

This tendency of secondary sterility to augment the impact of abortion would be most strongly felt late in the reproductive period combined with no contraception. No contraception assures a high incidence of pregnancy and therefore abortion among the couples who are still fertile. Proximity to the end of the reproductive period means that secondary sterility commonly follows closely after abortion. Conversely, if contraception is cfficient, then there are few pregnancies and abortions; while early or even in the middle of the reproductive period, omsets of secondary sterility are rare. In these two cases the role of secondary sterility is necessarily much reduced.

A second bias of the present model is to exaggerate the impact of abortion when the starting age is young and contraception is highly efficient. As Table IV indicates, when contraception is efficient, the impact of abortion tends to decline as the starting age of woman rises. That is, under efficient contraception, the younger is the age of the woman, the greater is the impact of an abortion. The present model by positing that a young starter of abortion retains her youthful fecundity throughout her residual reproductive period yields more births averted per abortion than would be the case if her fecundity were allowed to decline as a natural function of ageing. Fairly surely, then, the second bias arises from the present model neglecting the agedependent changes in fecundability, risk of fetal wastage, and postbirth anovulation.

\section{APPENDIX I: Single Abortion Experiment}

An induced abortion does not alter the time of initiation of the pregnancy it terminates but may delay the next birth by necessitating one or more extra pregnancies. Three measures of impact per abortion are $H$, the number of additional pregnancies necessitated per abortion to attain another birth; $I$, the net delay of next birth per abortion; and $B$, births averted per abortion. These indices are taken up in turn.

Let $M$-pregnancies denote those pregnancies that in the absence of induced 
abortion would have ended as miscarriages. Analogously, $S$ - and $L$-pregnancies distinguish pregnancies potentially ending in stillbirths or live births.

Without induced abortion, the expected number of pregnancies $E(N)$ required to produce a live birth is $1 / \theta_{4}$ since $N$ may be taken as a random variable distributed according to the geometric distribution with parameter $\theta_{4}$. Each pregnancy is an independent trial with the probability of success (a pregnancy leading to a live birth) fixed at $\theta_{4}$.

Now consider $E\left(N^{\prime}\right)$, the expected number of pregnancies needed for a live birth predicated upon each woman deliberately aborting one pregnancy. Pending that induced abortion, the probability of a pregnancy ending in a miscarriage early enough to forestall an abortion is $h \theta_{2}$. A proportion $\left(1-\theta_{2} h\right)$ of the women, not experiencing such a miscarriage, will deliberately abort their first pregnancy and therefore need an average of $1+1 / \theta_{\mathbf{4}}$ pregnancies for a next birth. A proportion $h \theta_{2}\left(1-h \theta_{2}\right)$ of the women first experiencing an early miscarriage, will deliberately abort their second pregnancy, and therefore need $2+1 / \theta_{4}$ pregnancies on average for a next birth. More generally, a proportion $\left(h \theta_{2}\right)^{j-1}\left(1-h \theta_{2}\right)$ will deliberately abort their $j$-th pregnancy after $j-1$ consecutive early miscarriages and therefore require an average of $j+1 / \theta_{4}$ pregnancies for a live birth. Hence

$$
\begin{aligned}
E\left(N^{\prime}\right) & =\sum_{j=1}^{\infty}\left(h \theta_{2}\right)^{j-1}\left(1-h \theta_{2}\right)\left(j+1 / \theta_{4}\right) \\
& =1 / \theta_{4}+\sum_{j=1}^{\infty} j\left(h \theta_{2}\right)^{j-1}\left(1-h \theta_{2}\right) \\
& =1 / \theta_{4}+1 /\left(1-\theta_{2} h\right) \\
& =1 / \theta_{4}+H,
\end{aligned}
$$

and $E\left(N^{\prime}\right)-E(N)=H$.

We now turn to the net delay of next birth. In the control cohort, without induced abortion, the expected interval from origin birth to next birth is

$$
u_{40}+u_{01,4}+u_{14}=u_{44} .
$$

In the presence of a single abortion, the interval from origin birth to next conception is unchanged, having an expected length of $u_{40}+u_{01,4}$. There follows an average of $H-1$ recurrence times $T_{11}$ between conceptions, each such pregnancy interval containing a miscarriage early enough to forestall abortion. Each of these intervals has an expected length of $\left(u_{12}^{*}-\delta\right)+u_{20}+u_{01,2}$, the term $u_{12}^{*}-\delta$ denoting the mean length of pregnancies brief enough to forestall abortion. (More explicit expressions for $\delta$ and $u_{01, i}$ are given later.) Next comes that recurrence time $T_{11}$ that encompasses the induced abortion, 
its expected length being $Z+u_{20}+u_{01,2}$. Completing the total interval from origin birth to next birth is the interval from postabortion conception to next birth which has an expectation $u_{14}$, the same as in the absence of abortion since by assumption (10) induced abortion does not cause subfecundity. Collecting results, the difference in duration to next birth between experimental and control cohorts is

$$
\begin{aligned}
I & =(H-1)\left(\eta_{2}-\delta+u_{01.2}\right)+\left(Z+u_{20}+u_{01,2}\right) \\
& =H\left(\eta_{2}+u_{01,2}\right)-H h \theta_{2}+\left(Z-u_{12}^{*}\right) .
\end{aligned}
$$

It remains to consider how many births are averted by this net delay $I$ of the next birth. From previous work (Perrin and Sheps, 1964), the number of births to be expected during a reproductive period of $T$ months ( $T$ large) if the renewal process starts right after a birth is

$$
E\left[N_{4}(T) \mid S_{4}\right]=\frac{T}{u_{44}}+\frac{u_{44}^{(2)}+u_{44}}{2 u_{44}^{2}}-1,
$$

where $u_{44}$ and $u_{44}^{(2)}$ are first and second moments around the origin of the birth interval $T_{44}$.

Expected births per member of the control cohort may be expressed as

$$
E\left[N_{4}(T) \mid S_{4}\right]=1+E\left[N_{4}\left(T-u_{44}\right) \mid S_{4}\right]
$$

while for members of the experimental cohort, it is

$$
E\left[N_{4}(T) \mid S_{4}\right]=1+E\left[N_{4}\left(T-u_{44}-I\right) \mid S_{4}\right] .
$$

Evaluating both right-hand expressions and subtracting the latter from the former, we obtain after cancellation

$$
B=I / u_{44} .
$$

It has been shown (Barrett, 1969) that the data of French and Bierman concerning length of gestation ending in spontaneous fetal loss (exclusive of stillbirths) are adequately fitted by a truncated geometric distribution with parameter .45. The proportion of miscarriages of length $i$ months (measured from last menstrual period) is $\phi(i)=C(0.45)(0.55)^{i-2}$, where $i=2,3, \ldots, 7$ and $C=1 /\left(1-0.55^{6}\right)$. The resultant mean length $u_{12}^{*}$ of pregnancy preceding miscarriage in the absence of induced abortion is 3.12.

In the presence of repeated abortion performed $Z$ months after LMP, the expected length of pregnancies ending in miscarriage is, by assumption (9),

$$
\begin{aligned}
u_{12}^{*}-\delta & =\frac{1}{h}\left(\sum_{i=2}^{Z-1} i \phi(i)+\frac{Z \phi(Z)}{2}\right), & & Z=3,4, \ldots, 7, \\
& =2, & Z & =2 .
\end{aligned}
$$


From an earlier analysis (Potter, 1970a),

$$
u_{01, i}=k u_{01,0}+(1-k)\left(\frac{1-f}{f}\right), \quad i=2,3,4,
$$

where

$$
k=\left(1-d^{\prime}\right)^{u_{i 0}} \quad \text { and } \quad u_{01.0}=\frac{1-p}{d+p}+\frac{d}{d+p}\left(\frac{1-f}{f}\right) .
$$

In addition,

$$
u_{44}=\frac{1}{\theta_{4}}\left\{\sum_{i=2}^{4} \theta_{i}\left(u_{01, i}+\eta_{i}\right)\right\}
$$

Since $u_{40}>u_{30}>u_{20}>0$, then provided only that $\mathrm{d}^{\prime}>0$ and $e>0$,

$$
u_{01,4}<u_{01,3}<u_{01,2}<u_{01,0} .
$$

Note that as the efficiency of contraception increases, i.e., as $d^{\prime} \rightarrow 0$ and $e \rightarrow 1.0$, the ratios $u_{01,3} / u_{01,2}$ and $u_{01,4} / u_{01,2}$ approach 1 . We may now consider the limiting impact of induced abortion as the efficiency of contraception progressively improves.

$$
\begin{aligned}
B^{-1} & ={ }_{I}^{u_{44}}=\frac{\frac{1}{\theta_{4}}\left\{\sum_{i=2}^{4} \theta_{i}\left(u_{01, i}+\eta_{i}\right)\right\}}{H\left\{u_{01,2}+\left(\eta_{2}-h \theta_{2} \delta+H^{-1}\left(Z-u_{12}^{*}\right)\right)\right\}} \\
& =\frac{\left\{\sum_{i=2}^{4} \theta_{i}\left(u_{01, i}+\eta_{i}\right)\right\}}{\theta_{4} H\left(u_{01,2}+C\right)} .
\end{aligned}
$$

As $d^{\prime} \rightarrow 0.0$ and $e \rightarrow 1.0$, only the values $u_{01, i}$ increase with the result that for $i=2,3,4$,

$$
\frac{u_{01, i}+\eta_{i}}{u_{01,2}+C} \rightarrow 1
$$

and therefore

$$
B^{-1} \rightarrow \frac{1}{\theta_{4} H}\left(\theta_{2}+\theta_{3}+\theta_{4}\right)=\frac{1}{\theta_{4} H} .
$$

Finally, as a check and for its own interest, we may derive an alternative expression for $I$ by conditionalizing on the potential outcome of pregnancies deliberately aborted. Let $R_{i}{ }^{*}$ denote the net delay of next birth per abortion conditioned to the potential outcome of the aborted pregnancy bcing outcome $i$. The relative frequencies of aborted pregnancies having potential outcomes 2, 3, and 4 are $H \theta_{2}(1-h), H \theta_{3}$, and $H \theta_{4}$, respectively. 
We have

$$
\begin{aligned}
R_{3}^{*} & =\left(Z+u_{20}+u_{01,2}\right)-\left(u_{13}^{*}+u_{30}+u_{01,3}\right) \\
& =-\left(\eta_{3}-\eta_{2}\right)-\left(u_{01,3}-u_{01,2}\right)+\left(Z-u_{12}^{*}\right) \\
& =R_{3}+\left(Z-u_{12}^{*}\right), \\
R_{4}^{*} & =Z+u_{20}+u_{01,2}+u_{14}-u_{14}^{*} \\
& =\eta_{2}+u_{01,2}+u_{14}-u_{14}^{*}+\left(Z-u_{12}^{*}\right) \\
& =R_{4}+\left(Z-u_{12}^{*}\right), \\
R_{2}^{*} & =Z+u_{20}+u_{01,2}-\left(Z+g^{*}+u_{20}+u_{01,2}\right) \\
& =-g^{*} \\
& =\left(Z-u_{12}^{*}\right)-g,
\end{aligned}
$$

where $g^{*}$ is the mean shortening by abortion of pregnancies fated otherwise to have ended in miscarriage and $g=g^{*}+\left(Z-u_{12}^{*}\right)$. More explicitly,

$$
g^{*}=\frac{\sum_{j=1}^{7-Z} j \phi(Z+j)}{\frac{1}{2} \phi(Z)+\sum_{j=1}^{7-Z} \phi(Z+j)} .
$$

By a tedious but straightforward argument, it can be shown that

$$
H \theta_{3} R_{3}+H \theta_{4} R_{4}=H\left(\eta_{2}+u_{01,2}\right)
$$

and

$$
\begin{aligned}
I & =H \theta_{2}(1-h) R_{2}{ }^{*}+\theta_{3} R_{3}{ }^{*}+\theta_{4} R^{*} \\
& =H\left(\eta_{2}+u_{01,2}\right)+\left(Z-u_{12}^{*}\right)-H(1-h) \theta_{2} g \\
& =H\left(\eta_{2}+u_{01,2}\right)+\left(Z-u_{12}^{*}\right)-H h \theta_{2} \delta,
\end{aligned}
$$

in agreement with earlier results.

\section{APPENDIX II: RePEATED ABortion ExPERIMENT}

If women of the experimental cohort practice repeated abortion with timing $Z$, then a proportion $\theta_{2} h$ of their pregnancies end in miscarriage and a proportion $1-\theta_{2} h$, or $H^{-1}$, end in induced abortion. The expected length of the former pregnancies is $u_{12}-\delta$; that of the latter $Z$. The expected recurrence time 
between pregnancy terminations (exclusive of the initial pregnancy interval between origin birth and next delivery) is

$$
u_{22}=\theta_{2} h\left(u_{01,2}+u_{12}^{*}-\delta+u_{20}\right)+\left(1-\theta_{2} h\right)\left(u_{01,2}+Z+u_{20}\right) .
$$

During a residual reproductive period of $T$ months ( $T$ large), the expected number of induced abortions is

$$
H^{-1} E\left[N_{2}(T) \mid S_{4}\right]=H^{-1}\left\{\frac{T}{u_{22}}+\frac{u_{22}^{(2)}+u_{22}}{2 u_{22}^{2}}-\frac{u_{42}}{u_{22}}\right\} .
$$

Respecting the control cohort, the number of births to be expected in the absence of abortion is

$$
E\left[N_{4}(T) \mid S_{4}\right]=\frac{T}{u_{44}}+\frac{u_{44}^{(2)}+u_{44}}{2 u_{44}^{2}}-1 .
$$

If we let $T \rightarrow \infty$, then the ratio of births prevented to induced abortions converges to

$$
\lim _{T \rightarrow \infty} \frac{E\left[N_{4}(T) \mid S_{4}\right]}{H^{-1} E\left[N_{2}(T) \mid S_{4}\right]} \rightarrow \frac{H u_{22}}{u_{44}}=\frac{I}{u_{44}} .
$$

The last equality follows because

$$
\begin{aligned}
H u_{22}= & H \theta_{2} h\left(u_{01,2}+u_{12}^{*}+u_{20}-\delta\right) \\
& +H\left(1-\theta_{2} h\right)\left(u_{01,2}+u_{12}^{*}+u_{20}+Z-u_{12}^{*}\right) \\
= & H\left(u_{01,2}+u_{12}^{*}+u_{20}\right)-\delta H \theta_{2} h+H\left(H^{-1}\right)\left(Z-u_{12}^{*}\right) \\
= & I .
\end{aligned}
$$

\section{ACKNOWLEDGMENTS}

The writer gratefully acknowledges support received from the Ford Foundation and The Population Council, the programming assistance of Barbara McCann and Joan Kieper, and the helpful comments of Nathan Keyfits, Mindel Sheps, and three anonymous reviewers.

\section{REFERENCES}

Barrett, J. C. 1969. A Monte Carlo simulation of human reproduction, Genus 25, 1-22. DAvis, K. 1963. The theory of change and response in modern demographic history, Population Index 29, 345-349. 
Hall, R. E. 1970. "Abortion in a Changing World," Vols. I and II, Columbia University Press, New York.

JAIN, A. K. 1969 a. Fetal wastage in a sample of Taiwanese women, Milbank Memorial Fund Quarterly 47, 297-306.

JaIn, A. K. 1969b. Pregnancy outcome and the time required for next conception, Populations Studies 23, 421-433.

JaIN, A. K. 1970. Demographic aspects of lactation and amenorrhea, Demography 7, 255-271.

JonEs, G. W. ANd Mauldin, W.P. 1967. Use of oral contraceptives with special reference to developing countries, Studies in Family Planning 24, 1-12.

KIRK, D. 1967. Prospects for reducing natality in the underdeveloped world, Annals 369.

Mauldin, W. P., Norman, D., and Stephan, F. F. 1967. Retention of IUD's: An international comparison, Studies in Family Planning 18, 1-12.

Murama'ss, M. 1960. Effect of induced abortion on the reduction of births in Japan, Milbank Memorial Fund Quarterly 38, 152-166.

Perrin, E. B. AND Sheps, M. C. 1964. Human reproduction: A stochastic process, Biometrics 20, 28-45.

PotTer, R. G. 1963. Birth intervals: structure and change, Population Studies 17, 162-164.

Potter, R. G. 1970a. Net delaying of next conception by contraception: A highly simplified case, Population Studies 24, 173-192.

PotTER, R. G. 1970b. Births averted by contraception: An approach through renewal theory, Theoretical Population Biology 1, 251-272.

Potrer, R. G. 1971. Inadequacy of a one-method family planning program, Studies in Family Planning 2, 1-6.

ShePs, M. C. 1964. Pregnancy wastage as a factor in the analysis of fertility data, Demography 1, 111-118.

Srinivasan, K. 1967. "Analytical Models for Two Types of Birth Intervals with Applications to Indian Population," unpublished Ph.D. dissertation, University of Kerala, Trivandrum, India. 